\title{
Die berufliche Integration von Jugendlichen mit Hauptschulbildung. Eine Längsschnittanalyse typischer Übergangsverläufe
}

In jüngster Zeit hat sich das Verhältnis von Angebot und Nachfrage auf dem Ausbildungsstellenmarkt entspannt. Jugendliche mit Hauptschulbildung haben allerdings nach wie vor große Schwierigkeiten bei der Suche nach einer Berufsausbildung und dem Einstieg in qualifizierte Erwerbsarbeit. Durch Maßnahmen des Übergangssystems oder den Verbleib an allgemeinbildenden Schulen versuchen sie, ihre Chancen auf dem Ausbildungsmarkt zu verbessern. Wie die Wege, die Sie dabei einschlagen, im Einzelnen aussehen und welche Bedingungen zum Erfolg oder zur Auskoppelung aus dem Berufsbildungssystem führen, ist Thema dieses Beitrags. ${ }^{\circ}$

BORIS GEIER

\section{Einleitung}

Klar strukturierte Übergangswege von der Schule in Ausbildung und Beruf, die einen nach Bildungsniveau differenzierten und reibungslosen Übergang ins Ausbildungs- und Erwerbssystem vorsehen, trifft man inzwischen bei immer weniger Jugendlichen an. Besonders betroffen von den Unwägbarkeiten beim Übergang von Schule in Ausbildung (und später dann von Ausbildung in Erwerbstätigkeit) sind Jugendliche mit Hauptschulbildung (Konsortium Bildungsberichterstattung 2006; Autorengruppe Bildungsberichterstattung 2008, 2010).

Anstatt direkt eine reguläre Berufsausbildung beginnen zu können, münden viele von ihnen in Bildungsgänge ein, die üblicherweise - geprägt durch den ersten nationalen Bildungsbericht (Konsortium Bildungsberichterstattung 2006) - unter dem Begriff „Übergangssystem“ zusammengefasst werden. Dieser von Krüger als „Chancenverbesserungssystem“ bezeichnete Sektor (Krüger 2004, S. 145) hat die Funktion, die Allgemeinbildung sowie berufliche und vorberufliche Kompetenzen zu verbessern, um so die $\mathrm{Zu}$ gangschancen zur regulären beruflichen Bildung zu erhöhen.

Weiterhin verbleibt eine wachsende Zahl von Hauptschulabsolventinnen und Hauptschulabsolventen im allgemeinbildenden Schulsystem. Dies kann Ausdruck einer Notlösung sein, um drohender Ausbildungslosigkeit zu entgehen. Es kann jedoch auch eine gezielte Strategie der schulischen Höherqualifizierung sein, um die beruflichen Chancen zu verbessern (Lex/Geier 2010; Geier et al. 2010). Und schließlich fallen viele Jugendliche gänzlich aus dem Berufsbildungssystem heraus und erfahren bereits früh nach der Beendigung der Hauptschule Phasen von Arbeitslosigkeit und prekärer Beschäftigung (Tillmann/Gehne 2012).

Sicher ist, dass Hauptschulabsolventinnen und Hauptschulabsolventen nach Beendigung ihrer Pflichtschulzeit vor unterschiedlichen Anschlussoptionen stehen, welche sich als mehr oder weniger realisierbar und chancenträchtig für die weitere berufliche Zukunft erweisen. Ziel dieses Beitrags ist es, die Wege, die diese Jugendlichen nach der Schule einschlagen, besser zu verstehen. Dazu werden typische Verläufe (bzw. Verlaufsmuster), wie sie sich nach dem Absolvieren der Hauptschule in einem Zeitraum von fünfeinhalb Jahren ergeben, beschrieben (Abschnitt 4.2). Anschließend wird der Frage nachgegangen, welche Faktoren Einfluss auf die Einmündung in unterschiedliche bildungs- und ausbildungsbezogene Karrierewege nehmen (4.3 und 4.4). Der empirischen Analyse vorangestellt ist ein kurzer Einblick in den Forschungsstand und die hier

- Die Erkenntnisse dieses Beitrags basieren auf einem von der Deutschen Forschungsgemeinschaft (DFG) geförderten Projekt (GZ: BR 3730/2-1). 
relevanten Fragestellungen (2) sowie in die Datenbasis (3) und das methodische Vorgehen (4.1). Abschnitt 5 fasst die Ergebnisse der Untersuchung zusammen.

\section{Forschungsstand und Fragestellungen}

Aus dem bisherigen Forschungsstand ergeben sich nur wenige Antworten auf die Frage, welche Bedingungen den Übergangsverlauf von der Schule ins Arbeitsleben für Jugendliche mit Hauptschulbildung beeinflussen. Insbesondere gibt es nur wenige aktuelle Studien, die Bildungsverläufe anhand von Längsschnittdaten analysieren und damit, über Zugangs- und Abgangszahlen hinaus, die zeitliche Struktur des Übergangsgeschehens abbilden.

Für das Verständnis der Bedingungen, die Übergangswege beeinflussen, liefern die retrospektiv angelegten Lebensverlaufsuntersuchungen des Max-Planck-Instituts für Bildungsforschung eine wichtige Quelle (Hillmert/Mayer 2004; Solga 2004, 2005; Wagner 2005). Unter anderem zeigen die Analysen, dass Jugendliche ohne bzw. mit geringwertigen Bildungsabschlüssen überwiegend in das Berufsbildungssystem (einschließlich des Übergangssystems) eintreten (Solga 2004, S. 52) und im Übergangssystem erworbene Schulabschlüsse die Chancen auf den Eintritt in eine reguläre Berufsausbildung erhöhen. Im Rahmen der wissenschaftlichen Begleitung des 1999 aufgelegten Sofortprogramms zum Abbau der Jugendarbeitslosigkeit (JUMP) wurden neben individuellen und Lebenslaufmerkmalen der teilnehmenden Jugendlichen auch Merkmale der regionalen Ausbildungs- und Arbeitsmärkte erhoben (Dietrich 2001). Nach den Ergebnissen dieser Studie verringern ungünstige Rahmenbedingungen auf den regionalen Ausbildungs- und Arbeitsmärkten und eine hohe Dauer der Teilnahme an arbeitsmarktpolitischen Maßnahmen die Chancen auf einen Wechsel in reguläre Ausbildung oder Erwerbsarbeit.

In der retrospektiv angelegten Längsschnittuntersuchung des Bundesinstituts für Berufsbildung (BIBB) (Beicht et al. 2008) wurden die Übergangsverläufe Jugendlicher vom Zeitpunkt des erstmaligen Verlassens einer allgemeinbildenden Schule rekonstruiert und somit auch in Hinblick auf ihre zeitliche Struktur untersucht. Mit einem ähnlich dem im vorliegenden Beitrag verwendeten Verfahren der Sequenzmusteranalyse wurden die nicht studienberechtigten Jugendlichen (mit maximal Mittlerem Schulabschluss) fünf Clustern von Übergangsmustern zugewiesen (ebd., S. 174). Ein direkter und dauerhafter Übergang in eine betriebliche Ausbildung gelang $38 \%$ der Jugendlichen. Verzögerte Übergänge in betriebliche Ausbildung mit zum großen Teil vorgeschalteten Aufenthalten im Übergangssystem wiesen $14 \%$ der Jugendlichen auf, $18 \%$ der Jugendlichen mündeten in eine nicht betriebliche Ausbildung, zum Teil direkt, zum Teil erst nach Wartezeiten, die sie überwiegend im Übergangssystem verbrachten. Bei einem Fünftel der Jugendlichen waren problematische Verläufe zu beobachten, welche nicht in Ausbildung führten oder nur nach langer Wartezeit in eine nicht betriebliche Ausbildungsform. Schließlich fand sich ein weitergehender dauerhafter Besuch von Fachoberschulen und Fachgymnasien bei $10 \%$ der Jugendlichen. Jugendliche mit maximal Hauptschulabschluss wiesen dabei ein höheres Risiko für verzögerte und nicht in Ausbildung bzw. schulische Höherbildung führende Verläufe auf. Jugendlichen mit Migrationshintergrund und jungen Frauen gelang der unmittelbare Einstieg in eine betriebliche Ausbildung seltener. Jugendliche mit Migrationshintergrund trugen zudem ein höheres Risiko für langwierige oder nicht gelingende Übergänge in Ausbildung (ebd., S. 178). Erfolgreiche Übergänge waren außerdem abhängig von der Wohnregion und dem Bildungs- und Berufsstatus der Eltern.

Beicht (2009, S. 4) kommt zu dem Ergebnis, dass $42 \%$ der Jugendlichen mit maximal Hauptschulabschluss an mindestens einer Maßnahme im Übergangssystem teilgenommen haben. Die Maßnahmeteilnehmenden wiesen einen ungünstigeren familiären Hintergrund auf und waren in der Schule weniger erfolgreich als Jugendliche, denen der Übergang in eine Ausbildung ohne einen vorherigen teilqualifizierenden Bildungsgang gelang.

Während die Längsschnittuntersuchung des BIBB Jugendliche aller Schularten retrospektiv in den Blick nahm, werden im Folgenden die Übergangswege einer reinen Hauptschüler-Population (Jungen und Mädchen) anhand prospektiv erhobener Daten untersucht. Im Mittelpunkt stehen die Übergangsverläufe der Jugendlichen nach dem (erstmaligen) Verlassen der Hauptschule. Von Interesse ist: $\mathrm{Zu}$ welchen Anteilen gelingt der friktionslose Übergang in Ausbildung? Welche Zwischenschritte werden vollzogen und wie sieht die zeitliche Struktur der Übergangsverläufe aus? Nach einer Darstellung typischer Übergangsverläufe werden Einflussfaktoren auf das Übergangsgeschehen untersucht. Gibt es erkennbare Strategien, mit denen Jugendliche auf schlechte Bedingungen auf dem Ausbildungs- und Arbeitsmarkt reagieren? Welche Rolle spielen Merkmale wie Geschlecht oder Migrationshintergrund für erfolgreiche Übergänge? Es werden Risiko- und Schutzfaktoren in $\mathrm{Zu}$ sammenhang mit dem Herausfallen aus dem Bildungssystem bzw. die erfolgreiche Integration begünstigende Faktoren in den Arbeitsmarkt identifiziert.

\section{Datenbasis}

Datenbasis für die folgenden Analysen ist das Übergangspanel des Deutschen Jugendinstituts (DJI) (vgl. Reißig et al. 2008), eine auf sechs Jahre angelegte bundesweite Längsschnittuntersuchung zu den Bildungs-, Ausbildungs- und Erwerbswegen von Hauptschülerinnen und Hauptschülern. 
Die Basiserhebung, an der sich 3.922 Jugendliche aus 126 Schulen beteiligten, fand im März 2004 als Fragebogenerhebung im Klassenverband statt. Seitdem wurden zunächst halbjährliche und ab dem Jahr 2006 jährliche Folgebefragungen durchgeführt, die mittels computergestützter Telefoninterviews erfolgten (CATI). Bei der Basiserhebung befanden sich die befragten Jugendlichen im letzten Schulbesuchsjahr einer Hauptschule, bzw. in Hauptschulzweigen von Gesamtschulen und Sekundarschulen. ${ }^{2}$ Vollständige Verlaufsdaten bis zum letzten Erhebungszeitpunkt im November 2009, mittels derer Übergangsbiografien in einem Zeitfenster von 66 Monaten bzw. fünfeinhalb Jahren rekonstruiert werden können, liegen für 900 Jugendliche vor.

Um die Panelattrition zu kontrollieren, wurden Ausfallanalysen durchgeführt. Es zeigte sich, dass die Verteilung personengebundener Merkmale, wie soziodemografische Merkmale (insb. Migrationshintergrund und Geschlecht), die Ressourcenausstattung und schulische Leistungen, über die Erhebungswellen hinweg hinreichend stabil blieben. Den größten Einfluss auf ein frühzeitiges Ausscheiden aus dem Panel, und damit die bedeutsamste Quelle für Verzerrung der Stichprobe über die Zeit, hat die jeweilige soziale Situation („Platzierung“, vgl. Fußnote 3) der Jugendlichen. So nehmen Jugendliche signifikant seltener an einer Erhebungswelle teil, wenn sie sich im Jahr davor ohne Arbeit und Ausbildung und damit in einer prekären Lebenssituation befanden. Bei der Interpretation der Ergebnisse ist daher auf eine mögliche Unterschätzung der Anzahl prekärer Stationen bzw. Jugendlicher mit problematischen Biografien zu achten.

Erhoben wurden neben soziodemografischen Merkmalen Informationen zur schulischen und privaten Situation der Jugendlichen, Erfahrungen mit schulischer Förderung und Ausbildungsvorbereitung im letzten Schuljahr, Bewerbungstrainings und -aktivitäten sowie Bildungs- und Ausbildungspläne der Jugendlichen. Zentrale Gegenstände ab der dritten Erhebung waren die Bildungs- und Ausbildungsepisoden der Jugendlichen, die seit dem Ende des Schuljahrs in der Pflichtschule erfragt wurden. Diese Informationen liegen als episodisches Datenmaterial mit monatsgenauem Beginn- und Enddatum vor. Dabei wurden die folgenden Arten von Episoden unterschieden:

- Ausbildung: Vollqualifizierende betriebliche, schulische und überbetriebliche Ausbildungen;

- Berufsvorbereitung: Berufsvorbereitungsjahr (BVJ), Berufsgrundbildungsjahr (BGJ), berufsvorbereitende Maßnahme (BvB);

- Berufliche Schulen: Bildungsgänge an Berufsfachschulen und anderen beruflichen Schulen, die keinen beruflichen Abschluss vermitteln, nicht zur Hochschulreife (HSR) führen und zu keiner der bereits genannten Berufsvorbereitungen gehören;

- Allgemeinbildende Schulen - keine HSR: Bildungsgänge an allgemeinbildenden Schulen, die nicht zur Hochschulreife führen;
- Allgemeinbildende Schulen - HSR: Bildungsgänge an allgemeinbildenden und beruflichen Schulen, die zur Hochschulreife führen;

- Studium: Studium an einer (Fach-)Hochschule;

- Beschäftigung nach erfolgreich angeschlossener Ausbildung;

- Beschäftigung ohne vorherige abgeschlossene Berufsausbildung;

- unversorgt: Jugendliche, die sich weder im Berufsbildungssystem noch in sonstigen geschützten Systemen befinden und die keiner Erwerbstätigkeit nachgehen;

- Sonstiges: Praktika, Freiwillige Jahre und Wehr- oder Zivildienst.

Abweichend von der Definition der Nationalen Bildungsberichte werden hier Berufsvorbereitung und Bildungsgänge an Berufsfachschulen und anderen beruflichen Schulen, die zu keinem beruflichen Abschluss führen, nicht zu einer Kategorie „Übergangssystem“ zusammengefasst, weil die Kategorie „Berufliche Schulen“ zum großen Teil aus Bildungsgängen an Berufsfachschulen besteht, in denen der Mittlere Bildungsabschluss erworben werden kann und erworben wird.

\section{Empirische Ergebnisse}

\subsection{Methodisches Vorgehen}

In einem ersten Schritt werden die Bildungs-, Ausbildungsund Erwerbsbiografien der Jugendlichen in ihrem zeitlichen Ablauf betrachtet. Hierbei wird das in den Sozialwissenschaften mittlerweile gebräuchliche Verfahren der Sequenzmusteranalyse eingesetzt (vgl. Abbott 1995; Aisenbrey 2000; Erzberger/Prein 1997): Ausgehend von einer zeitlich geordneten Sequenz monatlicher Platzierungen ${ }^{3}$ (Status) der Jugendlichen werden Ähnlichkeiten (bzw. Unähnlichkeiten) der individuellen Verläufe zueinander mittels eines

(2) Wegen unterschiedlicher Regelungen in den Bundesländern befanden sich $37 \%$ der untersuchten Jugendlichen in der 10. Klassenstufe, $49 \%$ in der 9. Klassenstufe. Außerdem enthält der Datensatz Schülerinnen und Schüler, die sich in einer bayerischen Praxisklasse befanden. In diesen speziellen Klassen an Hauptschulen sind Jugendliche zusammengefasst, von denen erwartet wird, dass sie den Schulabschluss nicht erreichen werden und die über hohe Praxisanteile dennoch in eine Ausbildung vermittelt werden sollen.

(3) Die Platzierung bestimmt sich anhand der Angaben der Jugendlichen über die hauptsächliche Beschäftigung in Sachen Ausbildung und Arbeit. Parallele Episoden, wie etwa geringfüge Beschäftigung während einer Ausbildung, wurden erhoben, gehen in die vorliegende Auswertung jedoch nicht mit ein. 
Optimal-Matching-Verfahrens bestimmt. In einem zweiten Schritt bilden die so gewonnenen Ähnlichkeitsmaße die Grundlage für eine Clusteranalyse, die ähnliche Verlaufsmuster zu Typen gruppiert. ${ }^{\circledR}$ Aus den vollständigen Verläufen $(\mathrm{N}=900)$ ließen sich sechs übergeordnete Verlaufstypen identifizieren.

Die Darstellung der Ergebnisse erfolgt üblicherweise mittels eines Sequenzmusterplots, der die Platzierungen jeder einzelnen Person im Zeitverlauf, gelesen von links nach rechts, wiedergibt. Aus technischen Gründen wird in Abbildung 1 eine etwas schematisierte Darstellung verwendet, die einzelne Verläufe zu Blöcken zusammenfasst, deren Höhe der Anzahl Jugendlicher entspricht, die diesen Verlauf aufweisen. Kurze Zwischenepisoden werden nicht angezeigt. Finden in einem Verlaufstyp, wie etwa in dem der „Problematischen Verläufe", häufige Wechsel von Statuspassagen über die Zeit und zwischen Personen statt, wird diese Struktur durch vertikale Balken wiedergegeben, welche nicht den tatsächlichen Statussequenzen einzelner Personen entsprechen, sondern lediglich das Bild unsteter Verläufe innerhalb eines Verlaufstyps andeuten sollen (vgl. Hupka-Brunner et al. 2011; Gaupp et al. 2012).

Um zu prüfen, welche Faktoren die Einmündung in einen bestimmten Cluster beeinflussen, wurden verschiedene Prädiktoren (regionale Kontextfaktoren, soziodemografische Faktoren und individuelle Faktoren) nacheinander blockweise in ein multinomiales logistisches Regressionsmodell zur Vorhersage der Zugehörigkeit zu einem der sechs Verlaufstypen eingegeben. Aus Gründen der einfachen Darstellung wird nur das Gesamt-Modell ausgewiesen, in dem alle Faktoren gleichzeitig untersucht worden sind (vgl. Abschnitt 4.3, Tabelle 1). Der Verlaufstyp „Direkteinstieg in Ausbildung“ diente dabei als Referenzkategorie der abhängigen Variablen, um friktionslose Übergänge an der ersten Schwelle mit alternativen Übergangsverläufen zu kontrastieren.

Die Koeffizienten werden als odds-ratios ausgegeben. Werte größer 1 deuten auf einen positiven Zusammenhang zwischen dem Prädiktor und der Zugehörigkeit zu einem Verlaufsmuster hin, Werte kleiner 1 stehen entsprechend für eine verringerte Wahrscheinlichkeit, einem Verlaufsmuster anzugehören, wenn ein bestimmtes Merkmal gegeben ist bzw. bei metrischen Daten, wenn sich die Maßzahl des Prädiktors um eine Einheit erhöht.

\subsection{Typische Verlaufsmuster}

Mit Hilfe des oben dargestellten Verfahrens wurden sechs typische Verlaufsmuster identifiziert, die sich auf die Zeitspanne von fünfeinhalb Jahren nach (erstmaliger) Beendigung der Hauptschulzeit beziehen (Abbildung 1).

(1) Der erste der sechs Verlaufstypen, „Direkteinstieg in Ausbildung", dem $26 \%$ der Jugendlichen zugeordnet sind, fasst Verläufe zusammen, die durch einen direkt nach der Hauptschule erfolgten Übergang in Ausbildung und daran anschließende Erwerbstätigkeit gekennzeichnet sind. Die
Ausbildung wurde vom Großteil der Jugendlichen stabil und erfolgreich durchlaufen. Eine geringe Zahl der Jugendlichen, die diesem ersten Verlaufstyp zugeordnet wurden, befindet sich nach wie vor in Ausbildung, darunter etwa hälftig Jugendliche mit einer Zweitausbildung und Jugendliche, die nach einer abgebrochenen Ausbildung erneut in Ausbildung sind. Außerdem werden Jugendliche, welche nach einem Jahr ohne institutionelle Versorgung in Ausbildung kommen, diesem Verlaufstyp zugeordnet (diese erscheinen aufgrund ihrer geringen Fallzahl nicht in Abbildung 1). Etwa $10 \%$ (1\% von allen) der Jugendlichen aus der Gruppe der Direkteinsteiger finden nach ihrer Berufsausbildung keinen Anschluss und sind für die restliche Dauer der Beobachtungsphase unversorgt.

(2) Der zweite Verlaufstyp, "Berufsvorbereitung - Ausbildung", dem $16 \%$ der Jugendlichen zugeordnet wurden, enthält Verläufe, die nach einer im Anschluss an die Schulzeit begonnenen Berufsvorbereitung (BVJ, BGJ oder BvB) in Ausbildung münden. Zu drei Vierteln (76 \%) handelt es sich dabei um einjährige Phasen in Berufsvorbereitung, $18 \%$ der Jugendlichen dieses Verlaufstyps münden nach zwei Jahren Berufsvorbereitung in Ausbildung. Ein mit 6 \% (1 \% von allen Jugendlichen) relativ geringer Anteil von Jugendlichen beginnt die Ausbildungsphase nach drei Jahren Berufsvorbereitung. Der Beginn einer Berufsausbildung findet dabei nicht in allen Fällen direkt im Anschluss an die Berufsvorbereitung statt, sondern erfolgt manchmal nach einer einjährigen, selten auch nach einer mehrjährigen Phase der Unversorgtheit. Langfristig gelingt etwa $50 \%$ der Jugendlichen dieses Verlaufstyps der Übergang in Erwerbsarbeit. Die restlichen Jugendlichen befinden sich noch in Ausbildung (35\%) oder sind nach Beendigung einer Ausbildung in eine längere Phase ohne beruflichen Anschluss eingetreten (15\%).

(3) Im dritten Verlaufstyp, „Berufliche Schulen - Ausbildung“" den $6 \%$ aller Jugendlichen aufweisen, finden sich Hauptschulabsolventinnen und Hauptschulabsolventen, die einen nicht vollqualifizierenden und nicht zur Hochschulreife führenden Bildungsgang an einer beruflichen Schule beginnen und anschließend in Ausbildung übergehen. Die Verweildauer in den beruflichen Schulen beträgt für etwa die Hälfte der Jugendlichen zwei Jahre, für jeweils ein Vier-
(4) Das Optimal-Matching-Verfahren wurde mit konstanten Kosten für alle Statuswechsel durchgeführt (indel=1, substitution =2). Die anschließende hierarchische Clusteranalyse wurde mit dem Fusionsalgorithmus von Ward durchgeführt. Zur Ermittlung der optimalen Anzahl der Cluster wurde auf numerische Kriterien verzichtet, stattdessen wurden die resultierenden Lösungen der Reihe nach, angefangen mit der 3-Clusterlösung bis hin zu der 12-Clusterlösung, als Sortierkriterium eines Sequenzplots verwendet. Aus den so sortierten und gruppierten Sequenzen wurde nach inhaltlichen Aspekten eine 6-Clusterlösung ausgewählt. 
tel ein Jahr oder drei Jahre. Die Dauer des Verbleibs an beruflichen Schulen macht eine Analyse der Bewältigung der zweiten Schwelle innerhalb des Beobachtungszeitraums nicht für alle Jugendlichen möglich. Knapp ein Drittel ist bis zum Ende des Beobachtungszeitraums nach Abschluss der Ausbildung in Beschäftigung übergegangen, die restlichen Jugendlichen befinden sich noch in Ausbildung. Auch in diesem Cluster gibt es Fälle mit meist einjährigen Phasen von Unversorgtheit zwischen beruflicher Schule und dem Eintritt in eine Berufsausbildung.

(4) Der vierte Verlaufstyp, „Allgemeinbildende Schule - Ausbildung", fasst $19 \%$ aller Jugendlichen zusammen, die nach dem Besuch einer weiteren allgemeinbildenden Schule eine Ausbildung begonnen haben. Mehr als die Hälfte der Jugendlichen (55\%) absolvieren dabei ein weiteres Jahr an einer Schule, meist mit dem Ziel, einen Mittleren Bildungsabschluss zu erlangen. Gut ein Viertel der Jugendlichen (26\%) verbringt zwei Jahre und knapp ein Fünftel (19\%) dreijährige Phasen an weiteren Schulen, wobei Letztere sich überwiegend in Bildungsgängen befinden, die zur Hochschulreife führen. Für die Bewertung des Übergangs an der zweiten Schwelle sind auch hier wiederum nur die Fälle zu beurteilen, bei denen der Eintritt in Ausbildung spätestens im dritten Übergangsjahr stattgefunden hat. Etwa einem Drittel der Jugendlichen dieses Verlaufstyps ist der Übergang in eine Erwerbstätigkeit gelungen.

(5) Der fünfte Verlaufstyp, „Durchgehend Schule“ (9 \% aller Jugendlicher) beschreibt Jugendliche, die sich während der gesamten Beobachtungszeit fast ausschließlich in schulischen Bildungsgängen befinden. Knapp zwei Drittel beginnen diesen Weg an einer allgemeinbildenden Schule, wo sie den Mittleren Bildungsabschlusses erwerben, und knapp ein Drittel direkt mit einem Bildungsgang, der zur Hochschulreife führt.

Episoden in beruflichen Schulen, die nicht zur Hochschulreife führen und zum Übergangssystem gerechnet werden, finden sich in diesem Verlaufstyp nur vereinzelt. Knapp $60 \%$ der Jugendlichen dieses Verlaufstyps (5\% von allen) studieren im sechsten Jahr nach dem Verlassen der Hauptschule an einer Hochschule. Erwähnt sei, dass sich die Gruppe der Studierenden zum Großteil (69 \%) aus Jugendlichen zusammensetzt, die die Hauptschule bis zur 10. Klassenstufe besucht hatten (55 \% an einer regulären Hauptschule, $43 \%$ an einem Hauptschulzweig einer Gesamtschule und $2 \%$ an einer Sekundarschule). Gut 50 \% dieser Jugendlichen hatten am Ende Ihrer Pflichtschulzeit bereits einen Mittleren Schulabschluss. Der Erfolg der restlichen 40 \% dieses Verlaufstyps, welche sich nach wie vor im Schulsystem befinden, kann noch nicht abschließend beurteilt werden.

(6) Im sechsten Verlaufstyp, „Problematische Verläufe“, der knapp ein Viertel (24 \%) der Jugendlichen ausmacht, werden Verläufe zusammengefasst, die durch früh eintretende und sich über mehrere Jahre erstreckende Phasen von Be-
ABB. 1

\section{Stationen Jugendlicher nach dem Verlassen der Hauptschule}

Schematisierte Darstellung der Verlaufstypen

$\begin{array}{llll}\square & \text { Ausbildung } & \text { Berufsvorbereitung } & \text { Berufliche Schule } \\ \square & \text { allg. bild. Schule ohne HSR } & \text { allg. bild. Schulen HSR } & \text { Studium } \\ \square & \text { Beschäftigung nach abge- } & \text { Beschäftigung ohne } & \square \text { unversorgt } \\ \text { schlossener Berufsausbildung } & \text { abgeschl. Berufsausbildung } & \end{array}$

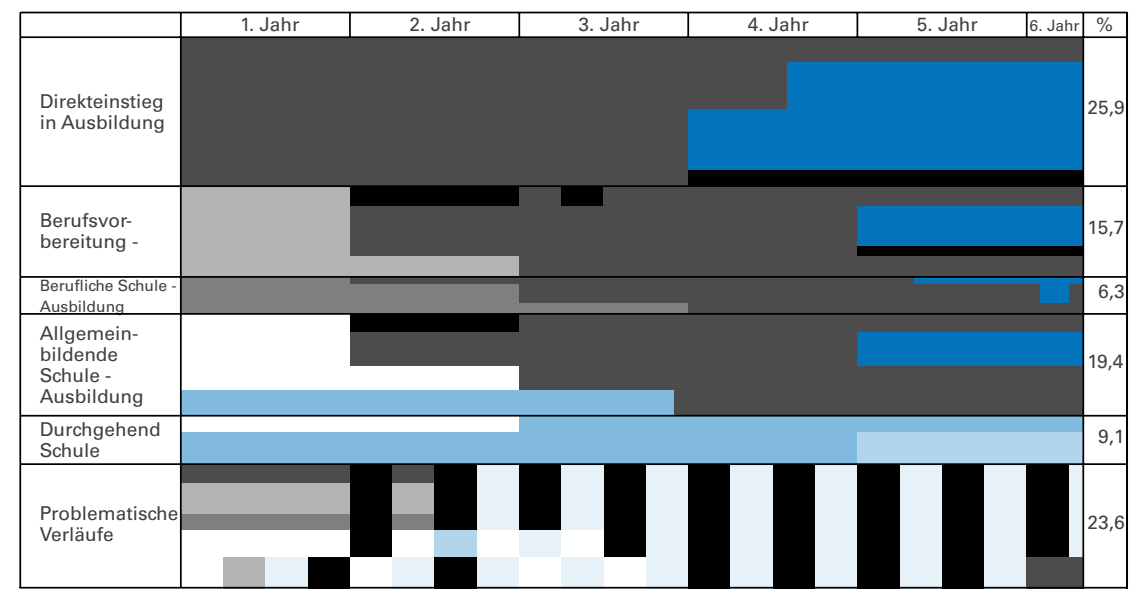

Quelle: Deutsches Jugendinstitut, eigene Darstellung.

schäftigungslosigkeit im häufigen Wechsel mit unqualifizierter Arbeit gekennzeichnet sind. Relativ wenige Jugendliche, etwa $15 \%$ (3\% von allen), sind dabei von Beginn an unversorgt. Die meisten folgen zunächst einer der aus den anderen Verlaufstypen bekannten Bahnen des Bildungsund Ausbildungssystems. So sind $16 \%$ der Jugendlichen mit problematischen Verläufen nach der Hauptschule direkt in Ausbildung eingemündet, haben diese aber abgebrochen und von da an keine weitere Möglichkeit zur beruflichen Integration nutzen können. Am Beginn der problematischen Entwicklung von mehr als einem Drittel der Jugendlichen (35\%) stand eine Berufsvorbereitung, weitere $11 \%$ wechselten nach der Hauptschulzeit in einen nicht vollqualifizierenden Bildungsgang einer beruflichen Schule. Ein weiteres gutes Viertel besuchte eine allgemeinbildende Schule oder Schulen, die zur Hochschulreife führen. Gut ein Fünftel der Jugendlichen dieses Verlaufstyps (5 \% von allen) gelingt es, im fünften und im sechsten Übergangsjahr noch in Ausbildung zu kommen, jedoch kann auch hier eine (positive) Prognose nur mit Zurückhaltung getroffen werden, da die Stabilität der Ausbildungsphase in Hinblick auf die in diesem Verlaufstyp häufig auftretenden Ausbildungsabbrüche zumindest fragwürdig ist.

\subsection{Determinanten der Verlaufsmuster}

Im Kern spiegeln die Verlaufstypen unterschiedliche $\mathrm{Zu}$ gänge zu einer Berufsausbildung, alternative Karrie- 
rewege (weiterer Schulbesuch und schulische Höherbildung) und als problematisch zu betrachtende Wege, die in dauerhafte Ausbildungslosigkeit münden (könnten), wider. Nun soll der Frage nachgegangen werden, ob und inwieweit diese typischen Übergangsverläufe nach dem Ende der Hauptschulzeit bereits vorgezeichnet sind und welche Faktoren die Wahrscheinlichkeit beeinflussen, einen bestimmten Karriereweg einzuschlagen. Zur Vorhersage der Verlaufsmuster wurden dabei Prädiktoren auf unterschiedlichen Ebenen herangezogen (Tabelle 1).

(1) Als Regionale Kontextfaktoren wurden die AngebotsNachfragerelationen (ANR) bezogen auf Ausbildungsplätze (ANR), die Arbeitslosenquote Jugendlicher unter 25 Jahren und die Quote von Ausbildungsplatzstellenbewerbern pro 100 Schulabgänger einbezogen. Neben diesen Einzelindikatoren wurde mit den sogenannte „Vergleichstypen der Arbeitsagenturen“" (Blien/Hirschenauer 2005) ein durch Typenbildung konstruierter Indikator verwendet, der allgemeine regionale Strukturmerkmale (z. B. großstädtisch vs. ländlich) mit arbeitsmarktrelevanten Merkmalen (Arbeitslosigkeit und deren Dynamik) verbindet. Der erste Typ dieser Klassifizierung „Bezirke i.d.R. in Ostdeutschland mit dominierendem Arbeitsplatzdefizit" deckt sich bei den vorliegenden Daten dabei mit dem Indikator Ostdeutschland, auf welchen deshalb verzichtet wurde.

(2) Neben den soziodemografischen Faktoren Geschlecht und Migrationshintergrund ${ }^{\oplus}$ wurden als familiale Faktoren das Merkmal „Alleinerziehendenhaushalt“, der höchste Schulabschluss und der höchste berufliche Status der Eltern (HISEI) einbezogen sowie ein Indikator für die elterliche Unterstützung bei berufsbezogenen Fragen.

(3) Als schulische Faktoren wurden einbezogen: der Schulabschluss nach Beendigung des letzten Pflichtschuljahres, der Mittelwert der Schulnoten in den Fächern Deutsch und Mathematik im letzten Pflichtschuljahr, Klassenwiederholungen, ein Indikator zum unentschuldigtem Absentismus („Schwänzverhalten“) sowie Skalen zur Schul- und Lernmotivation.

(4) Schließlich werden im Block individuelle Faktoren verschiedene soziale und psychische Merkmale der Jugendlichen kontrolliert. Hierzu zählen die Aktivität in Vereinen, die Anzahl enger Freunde sowie Skalen zur Messung von sozialer Kompetenz, Depression, Selbstwert, Selbstwirksamkeit und Zukunftspessimismus.

\subsection{Ergebnisse und Diskussion}

Mit den regionalen Kontextfaktoren wurden Gegebenheiten des Ausbildungs- und Arbeitsmarkts auf ihre Bedeutung für die weiteren Karrierewege der Jugendlichen kontrolliert. Für die prominenteste Differenzierung arbeitsmarktbezogener Gelegenheitsstrukturen, dem Vergleich zwischen
Ost- und Westdeutschland, zeigen sich Unterschiede vor allem in Hinblick auf problematische Verläufe (vgl. hier wie zu den folgenden Ergebnissen Tabelle 1). Verglichen mit dem Vergleichstyp der Arbeitsamtsbezirke in Westdeutschland mit durchschnittlicher Arbeitslosigkeit finden sich im Osten verringerte Risiken für problematische Verläufe. Diese werden jedoch erst nach dem Einbezug familialer und individueller Faktoren statistisch bedeutsam, was auf eine kompensatorische Funktion des ostdeutschen Ausbildungssystems schließen lässt. Letztere wird zu einem Gutteil durch das deutlich bessere Angebot vor allem überbetrieblicher Ausbildungsplätze im Osten möglich: In überbetriebliche Ausbildungen münden im Osten etwa $15 \%$ der Jugendlichen, im Westen sind es nur etwa $3 \%$ (siehe auch den Beitrag von Ulrich in diesem Heft). Weitere differenzierende Muster im Vergleich zwischen Ost- und Westdeutschland, etwa was die Wege über Berufsvorbereitung und Schulen anbelangt, sind zunächst nicht festzustellen. Weitergehende belastbare Aussagen über Ost-West Unterschiede sind aufgrund der geringen Fallzahlen Jugendlicher mit Wohnsitz im Osten im Datensatz allerdings auch nicht möglich.

Ein weiterer Effekt, der den Typisierungen der Arbeitsamtsagenturen entnommen werden kann, ist der deutliche Trend zur schulischen Höherbildung in großstädtisch geprägten Bezirken in Westdeutschland mit hoher Arbeitslosigkeit. Zentren in Westdeutschland mit günstiger Arbeitsmarktlage weisen hingegen einen gegenläufigen Trend auf. Jugendliche, die in diesen Regionen leben, haben im Vergleich zu Jugendlichen in westdeutschen Bezirken mit durchschnittlicher Arbeitslosigkeit eine signifikant geringere Wahrscheinlichkeit, in den Verlaufstyp „Durchgehend Schule“ einzumünden. Da dieser Effekt bei alleiniger Kontrolle der Arbeitslosenzahlen nicht auftritt, ist zunächst von einem Phänomen auszugehen, das aus einer Verknüpfung der Lage auf dem Ausbildungs- und Arbeitsmarkt mit weiteren regionsspezifischen Komponenten (Angebot und Dichte weiterführender Schulen, kollektive Trends in Großstädten) besteht und die Entscheidung für oder gegen weiteren Schulbesuch beeinflusst.

Mit der regionalen Arbeitslosenquote erhöht sich die Wahrscheinlichkeit für Verläufe, die über Berufsvorbereitung oder berufliche Schulen in Ausbildung führen. Der Effekt tritt für beide Verlaufstypen erst nach Einbezug schulischer Faktoren ein und ist für den Verlaufstyp „Berufliche Schulen - Ausbildung" stärker ausgeprägt. Ein Effekt der Knappheit von Ausbildungsstellen zeigt sich erst, wenn die

(5) Als Jugendliche mit Migrationshintergrund wurden definiert: Jugendliche, die nicht in Deutschland geboren sind und/oder deren Eltern bzw. ein Elternteil nicht in Deutschland geboren sind und/oder wenn zuhause (auch) eine andere Sprache als deutsch gesprochen wird und/oder wenn Jugendliche eine andere als die deutsche Staatsangehörigkeit besitzen. 


\section{Regressionsmodelle zur Vorhersage des Verlaufstyps (Gesamtmodell)}

\begin{tabular}{|c|c|c|c|c|c|}
\hline & $\begin{array}{l}\text { Berufsvorbe- } \\
\text { reitende (BV) - } \\
\text { Ausbildung }\end{array}$ & $\begin{array}{l}\text { Berufliche Schule } \\
\text { - Ausbildung }\end{array}$ & $\begin{array}{l}\text { Allgemein- } \\
\text { bildende Schule - } \\
\text { Ausbildung }\end{array}$ & $\begin{array}{l}\text { Durchgehend } \\
\text { Schule }\end{array}$ & $\begin{array}{l}\text { Problematische } \\
\text { Verläufe }\end{array}$ \\
\hline \multicolumn{6}{|l|}{ Regionale Kontextfaktoren } \\
\hline \multicolumn{6}{|l|}{$\begin{array}{l}\text { Vergleichsgruppen AA (Ref: Bez WD mit } \\
\text { durchschnittlicher Arbeitslosigkeit) }\end{array}$} \\
\hline - Bezirke idR in OD mit dom. Arbeitsplatzdefizit & 0,59 & 1,92 & 0,50 & 0,61 & $0,17^{*}$ \\
\hline - Großstädtisch WD mit hoher Arbeitslosigkeit & 0,70 & 0,62 & 1,66 & $10,75^{*}$ & 1,34 \\
\hline - Zentren WD mit günstiger Arbeitsmarktlage & 0,81 & 2,50 & 0,82 & $0,05^{*}$ & $0,34^{*}$ \\
\hline $\begin{array}{l}\text { - Bez WD mit guter Arbeitsmarktlage und } \\
\text { hoher Dynamik }\end{array}$ & 1,23 & $3,95+$ & 0,84 & 0,42 & 0,51 \\
\hline Arbeitslosenquote U20 (2005) & $1,21+$ & $1,58 * *$ & 1,09 & 0,86 & 1,11 \\
\hline ANR 2005 & $1,13+$ & $1,26^{*}$ & 1,04 & 1,07 & 1,08 \\
\hline Ausb.-Stellenbewerber pro 100 Schulabgänger & 1,00 & 0,96 & 0,98 & 1,03 & 1,01 \\
\hline \multicolumn{6}{|l|}{ Soziodemografie } \\
\hline Männlich & 0,99 & 1,09 & 0,93 & $0,50+$ & 0,85 \\
\hline $\mathrm{MH}$ & $1,76+$ & 1,98 & 1,23 & $2,16^{*}$ & $2,63 * *$ \\
\hline \multicolumn{6}{|l|}{ Familiale Faktoren } \\
\hline Alleinerziehendenhaushalt & 1,25 & 0,73 & 1,08 & 1,41 & 1,59 \\
\hline HISEI & 1,01 & 1,01 & 1,01 & $1,03+$ & 0,99 \\
\hline Höchster SA Eltern & 0,97 & 1,21 & $1,43+$ & $1,92^{*}$ & 1,33 \\
\hline Unterstützung durch Eltern & 0,74 & 0,78 & 1,14 & 0,87 & 0,87 \\
\hline \multicolumn{6}{|l|}{ Schulische Faktoren } \\
\hline Ohne HSA (Ref: HSA) & 1,63 & 0,51 & 1,94 & $2,46+$ & 1,73 \\
\hline MSA (Ref: HSA) & $0,26 * * *$ & $0,16^{* *}$ & $0,20 * * *$ & $0,42+$ & $0,17 * * *$ \\
\hline Schulnoten & 0,98 & $0,42 * *$ & $0,47 * * *$ & $0,57^{*}$ & $0,69+$ \\
\hline Klassenwiederholer & 0,80 & 0,97 & $0,34 * * *$ & $0,32 * *$ & 0,93 \\
\hline Schulschwänzen & 1,62 & 0,24 & 1,24 & 1,00 & $1,99+$ \\
\hline Schulmotivation & 1,04 & 0,63 & 1,60 & 1,21 & 0,67 \\
\hline Lernmotivation & $1,89+$ & $2,92^{*}$ & 0,92 & 1,61 & $2,01+$ \\
\hline \multicolumn{6}{|l|}{ Individuelle Faktoren } \\
\hline Aktiv in Vereinen & 0,85 & 1,27 & 0,69 & 0,57 & 0,63 \\
\hline Anzahl enger Freunde & 1,00 & 0,99 & 1,00 & 0,96 & 1,00 \\
\hline Soziale Kompetenz & 0,72 & $0,38^{*}$ & 0,74 & 0,82 & 0,72 \\
\hline Depression & 1,05 & 1,11 & 1,24 & 0,76 & 1,36 \\
\hline Selbstwert & 0,99 & 0,94 & 1,52 & 1,48 & 1,22 \\
\hline Selbstwirksamkeit & 1,02 & 0,89 & 1,19 & 0,76 & 0,85 \\
\hline Zukunftspessimismus & $2,02 *$ & 1,06 & $2,98 * * *$ & 1,32 & 1,28 \\
\hline $\begin{array}{l}\text { Nagelkerkes } \mathrm{R}^{2}=.37 \\
\mathrm{~N}=611\end{array}$ & & & & & WSI Mitteilungen \\
\hline
\end{tabular}

für den Zugang zu einer Berufsausbildung wichtigen Signale, wie Schulabschlüsse und Schulnoten, kontrolliert werden. Darüber hinaus kann festgestellt werden, dass die Wahrscheinlichkeit für einen Verlauf, der über den Besuch einer beruflichen Schule zur Ausbildung führt, mit besseren Schulnoten wächst. Der Besuch etwa einer Berufsfachschule ist also weniger eine Ausweichstrategie für schlechte Schülerinnen und Schüler, die aufgrund der schlechten Marksituation keine Ausbildungsstelle bekommen, sondern eher die bewusste Entscheidung guter Schülerinnen und Schüler, die in Antizipation der schlechten Marktsituation ihre „Marktposition“ durch den Erwerb weiterer Zertifikate verbessern wollen.

Die Ergebnisse in Hinblick auf die Angebots-NachfrageRelation (ANR) sind zunächst kontraintuitiv. Es zeigen sich signifikante Zusammenhänge zu den Verlaufstypen „Berufsvorbereitung - Ausbildung“ und „Berufliche Schulen - Ausbildung“, welche relativ stabil bzw. unabhängig vom Einbezug weiterer Faktoren bestehen. Jedoch vergrößert sich mit einer günstigeren Angebots-Nachfragerelati- 
on die Wahrscheinlichkeit, einen Weg in eine berufliche Ausbildung einzuschlagen, der über Zwischenschritte führt (im Vergleich zum Direkteinstieg). Eine mögliche Erklärung wäre die mangelnde Passung zwischen angebotenen Stellen und den Wünschen und Fähigkeiten der Hauptschulabsolventinnen und Hauptschulabsolventen. Dies würde auf die Notwendigkeit einer weiteren beruflichen Orientierung und (Teil-)Qualifizierung verweisen, welche die Jugendlichen in Berufsvorbereitung und an Berufsfachschulen anstreben. Anzumerken ist jedoch auch, dass die ANR umso günstiger ausfällt, je mehr Jugendliche einer Region Berufsvorbereitungen und berufliche Schulen besuchen, da diese dann nicht mehr auf der Nachfrageseite auftauchen. Eine günstige ANR wäre somit die Folge hoher Besuchsraten im Übergangssystem und nicht deren Erklärungsfaktor.

Bei den soziodemografischen Faktoren ist der starke Vorhersagewert des Merkmals Migrationshintergrund für die weiteren bildungs- und ausbildungsbezogenen Wege hervorzuheben. Für Jugendliche mit Migrationshintergrund ist (unter umfangreicher Kontrolle anderer Faktoren) die Wahrscheinlichkeit eines Weges über Berufsvorbereitung in Ausbildung sowie von problematischen Verläufen deutlich erhöht. Parallel dazu zeigt sich bei Jugendlichen mit Migrationshintergrund eine starke Präferenz für den weiteren Schulbesuch.

Das Geschlecht der Jugendlichen spielt bei der Betrachtung der Verlaufsmuster nur noch in Hinblick auf den Verlaufstyp „Durchgehend Schule“ eine Rolle, welcher bei männlichen Jugendlichen deutlich seltener auftritt. Andere Unterschiede scheinen sich bei der Betrachtung der Gesamtverläufe zunächst zu nivellieren.

Unter den familialen Faktoren besitzt lediglich der höchste Schulabschluss der Eltern einen bedeutsamen Vorhersagewert für die Wege der Jugendlichen. Je höher dieser Schulabschluss ist, desto eher schlagen die Jugendlichen Wege zu einer weiteren schulischen Qualifizierung („Allgemeinbildende Schule - Ausbildung“ und „Durchgehend Schule") ein.

Starke Effekte zeigen sich bei den schulischen Faktoren. So erhöht das Vorliegen eines Mittleren Bildungsabschlusses die Chancen auf einen direkten Übergang in Ausbildung um ein Vielfaches und verringert entsprechend die Wahrscheinlichkeit, einem der anderen Verlaufstypen anzugehören. Anders hingegen ist der Einfluss der Schulnoten. Ihnen kommt weniger ein positiver Effekt bei der Suche nach einer Ausbildungsstelle zu, relevant sind sie vielmehr, wenn ein Zugang zur weiteren Bildung gesucht wird. So steigt die Wahrscheinlichkeit für Wege über berufliche und allgemeinbildende Schulen sowie für Wege durchgehenden Schulbesuchs mit besseren Schulnoten. Vergleicht man die Wahrscheinlichkeit für einen Direkteinstieg in Ausbildung mit der für die Zugehörigkeit zum Verlaufstyp „Berufsvorbereitung - Ausbildung“', so zeigt sich deren Unabhängigkeit von den Schulnoten. Klassenwiederholer besuchen signifikant seltener weitere allgemeinbildende Schulen („allgemeinbildende Schulen - Ausbildung“ und „durchgehend
Schule“); „Schulschwänzen“ schließlich, erhöht das Risiko für den problematischen Verlaufstyp.

Die individuellen Faktoren besitzen insgesamt wenig Erklärungskraft für die Vorhersage der Verlaufsmuster.

\section{Fazit}

Betrachtet man die Verlaufsmuster insgesamt, lässt sich die Berufsausbildung als die dominierende Station innerhalb der ersten fünfeinhalb Jahre nach Abgang von einer Hauptschule erkennen. Friktionslose Übergänge an der ersten Schwelle weist jedoch nur etwa ein Viertel der Hauptschulabsolventinnen und Hauptschulabsolventen auf, deutlich weniger als etwa in der von Beicht et al. (2008) untersuchten Gruppe von Jugendlichen mit maximal Mittlerem Schulabschluss. Ein größerer Anteil Jugendlicher (42\%) mündet erst nach dem Absolvieren unterschiedlicher Zwischenschritte, wie dem Besuch weiterer allgemeinbildender oder beruflicher Schulen und der Teilnahme an einem berufsvorbereitenden Bildungsgang, in stabile Ausbildungsverläufe ein. Jugendliche, die nach dem Besuch einer beruflichen oder allgemeinbildenden Schule in Ausbildung münden, sind sich dabei ähnlicher als Jugendliche, die den Weg über eine klassische Berufsvorbereitung gehen. So sind es eher Schüler mit guten Schulnoten, die den schulischen Weg wählen, bzw. mit Blick auf die Aufnahmebedingungen auch wählen können. Eine Benachteiligung von Jugendlichen mit Migrationshintergrund spiegelt sich in einem erhöhten Risiko für Zwischenschritte in Berufsvorbereitung wider, das trotz Kontrolle der Schulnoten besteht. Dies kann als Hinweis darauf gewertet werden, dass Jugendliche mit Migrationshintergrund besonderen Diskriminierungsprozessen ausgesetzt sind.

Inwiefern die Art der Zwischenschritte den Erfolg langfristiger Integration in den Arbeitsmarkt beeinflusst, kann aufgrund der begrenzten zeitlichen Anlage der Studie nicht abschließend geklärt werden. Auswertungen zum weiteren Schulbesuch (Geier et al. 2010) zeigen jedoch, dass im Anschluss an weiteren Schulbesuch aufgenommene Ausbildungen tendenziell anspruchsvoller (gemessen am ISEI ${ }^{\circledR}$ ) sind.

Neben den „klassischen“ Verläufen, die mit oder ohne weitere Zwischenschritte eine zunächst sichere Einmündung in Ausbildung beinhalten, gehen etwa $9 \%$ der Jugendlichen den Weg von der Hauptschule zur Hochschulreife, eine Quote, die einerseits Zweifel an der Treffsicherheit der Zuweisungen am Ende der Primarstufe in Schulformen der Sekundarstufe I weckt. Andererseits zeigt sich hier die Mög-

6 ISEI ist ein Akronym für International Socio-Economic Index of Occupational Status (deutsch: internationales sozioökonomisches Maß des beruflichen Status). 
lichkeit einer Mobilität nach oben, welche allerdings zum Großteil von Jugendlichen genutzt wird, die die Hauptschule bis zur 10. Jahrgangsstufe besuchten.

Besondere Bedeutung ist dem Umstand zu widmen, dass knapp ein Viertel der Jugendlichen problematische bzw. nicht erfolgreiche Wege in die Ausbildung aufweist. Diese Verläufe sind durch lange Phasen in ungelernter Arbeit und in Arbeitslosigkeit gekennzeichnet. Risikofaktoren, die die Wahrscheinlichkeit einer Einmündung in diesen Verlaufstyp erhöhen, sind Migrationshintergrund, mangelnde Schulmotivation sowie häufiges „Schulschwänzen“. Darüber hinaus scheint es regionale Schutzfaktoren zu geben: So sind die Risiken einer Einmündung in problematische Verläufe in Zentren mit günstiger Arbeitsmarktlage und in Ostdeutschland deutlich geringer. Dennoch ist eine Vorhersage problematischer Verläufe an dieser Stelle schwierig und bedarf einer differenzierteren Betrachtung, in der die ersten Schritte in problematische Phasen genauer beleuchtet werden. Betrachtet man das erste Jahr im Muster dieser Verläufe, entsprechen die Platzierungen im Großen und Ganzen dem Muster der restlichen Verlaufstypen: So sind direkte Übergänge in Ausbildung ebenso wie Übergänge in weitere allgemeinbildende Schulen und in berufsvorbereitende Bildungsgänge zu beobachten. Nur relativ selten gibt es schon im ersten Jahr Platzierungen in prekären Stationen. Es liegt also der Schluss nahe, dass der Beginn problematischer Karrieren während oder nach Beendigung des ersten Schrittes nach der Hauptschule zu suchen ist. Einen genaueren Blick auf die Situation dieser ausgegrenzten Jugendlichen werfen Tillmann/Geh ne (2012).

Schließlich ist auf einen kleinen, aber nicht zu vernachlässigenden Prozentsatz von $5 \%$ der Jugendlichen hinzuweisen, dem der Übergang in Ausbildung zu einem relativ späten Zeitpunkt nach fünf bis sechs Jahren gelingt. Diesen Späteinsteigern bzw. Nachqualifizierern und den Bedingungen für die berufliche Integration Jugendlicher, die das System bereits für längere Zeit verlassen haben, ist angesichts der allgemeinen Entstrukturierung der Bildungsverläufe intensiver nachzugehen.

\section{LITERATUR}

Abbott, A. (1995): Sequence analysis: new methods for old ideas, in: Annual Review of Sociology 21 (1), S. 93-113

Aisenbrey, S. (2000): Optimal Matching Analyse, Opladen

Autorengruppe Bildungsberichterstattung (Hrsg.) (2008): Bildung in Deutschland 2008. Ein indikatorengestützter Bericht mit einer Analyse zu Übergängen im Anschluss an den Sekundarbereich II, Bielefeld

Autorengruppe Bildungsberichterstattung (Hrsg.) (2010): Bildung in Deutschland 2010. Ein indikatorengestützter Bericht mit einer Analyse zu Perspektiven des Bildungswesens im demografischen Wandel, Bielefeld

Baethge, M. (2008): Das Übergangssystem: Struktur - Probleme - Gestaltungsperspektiven, in: Münk, D./Rützel, J./Schmidt, C. (Hrsg.): Labyrinth Übergangssystem, Bonn, S. 53-67

Baethge, M./Solga, H./Wieck, M. (2007): Berufsbildung im Umbruch. Signale eines überfälligen Aufbruchs, Bonn

Beicht, U. (2009): Verbesserung der Ausbildungschancen oder sinnlose Warteschleife? BIBB Report 11/09, Bonn

Beicht, U./Friedrich, M./Ulrich, J. G. (Hrsg.) (2008): Ausbildungschancen und Verbleib von Schulabsolventen, Bonn
Blien, U./Hirschenauer, F. (2005): Welche Arbeitsagenturen sind vergleichbar?, in: Institut für Arbeitsmarkt- und Berufsforschung der Bundesagentur für Arbeit (Hrsg.): IAB Forschungsbericht (24), Nürnberg

Dietrich, H. (2001): Wege aus der Jugendarbeitslosigkeit - Von der Arbeitslosigkeit in die Maßnahme, in: Mitteilungen aus der Arbeitsmarkt und Berufsforschung 34 (4), S. 419-437

Erzberger, C./Prein, G. (1997): Optimal-Matching-Technik: Ein Analyseverfahren zur Vergleichbarkeit und Ordnung individuell differenter Lebensverläufe, in: ZUMA-Nachrichten 40 (21), S. 325-336

Gaupp, N./Geier, B./Hupka-Brunner S. (2012): Chancen bildungsbenachteiligter junger Erwachsener in der Schweiz und in Deutschland: Die (Nicht-)Bewältigung der 2. Schwelle, in: Zeitschrift für Soziologie der Erziehung und Sozialisation 32 (3), S. 299-318

Geier, B./Kuhnke, R./Reißig, B. (2010): Erfolgreiche Übergänge in Ausbildung durch verlängerten Schulbesuch? Ergebnisse des DJI-Übergangspanels, in: Krekel, E./Lex, T. (Hrsg.): Neue Jugend, neue Ausbildung? Beiträge aus der Jugend und Bildungsforschung, Bielefeld, S. 113-128

Greinert, W.-D. (2007): Kernschmelze - der drohende GAU unseres Berufsausbildungssystems, Berlin

Hillmert, S./Mayer, K. U. (Hrsg.) (2004): Geboren 1964 und 1971. Neuere Untersuchungen zu den Ausbildungs- und Berufschancen in Westdeutschland, Wiesbaden

Hupka-Brunner S./Gaupp, N./Geier, B./Lex, T./Stalder, B. (2011): Chancen bildungsbenachteiligter Jugendlicher: Bildungsverläufe in der Schweiz und in Deutschland, in: Zeitschrift für Soziologie der Erziehung und Sozialisation 31 (1), S. $62-78$

Konsortium Bildungsberichterstattung (Hrsg.) (2006): Bildung in Deutschland, Bielefeld

Krüger, H. (2004): Zur Datenlage vollzeitschulischer Berufsbildung, in: Baethge, M./Buss, K.-P./Lanfer, C. (Hrsg.): Expertisen zu den konzeptionellen Grundlagen für einen Nationalen Bildungsbericht - Berufliche Bildung und Weiterbildung/Lebenslanges Lernen, Berlin, S. 141-164

Lex, T./Geier, B. (2010): Übergangssystem in der beruflichen Bildung: Wahrnehmung einer zweiten Chance oder Risiken des Ausstiegs?, in: Bosch, G./ Krone, S./Langer, D. (Hrsg.): Das Berufsbildungssystem in Deutschland. Aktuelle Entwicklungen und Standpunkte, Wiesbaden, S. 165-185

Reißig, B./Gaupp, N./Lex, T. (Hrsg.) (2008): Hauptschüler auf dem Weg von der Schule in die Arbeitswelt, München

Solga, H. (2004): Ausgrenzungsgefahren trotz Integration - die Übergangsbiographien von Jugendlichen ohne Schulabschluss, in: Hillmert, S./Mayer, K. U. (Hrsg.): Geboren 1964 und 1971. Neuere Untersuchungen zu Ausbildungs- und Berufschancen in Westdeutschland, Wiesbaden, S. 39-64

Solga, H. (2005): Ohne Abschluss in die Bildungsgesellschaft, Opladen Tillmann, F./Gehne, C. (2012): Situation ausgegrenzter Jugendlicher. Expertise unter Einbeziehung der Perspektive der Praxis. Bundesarbeitsgemeinschaft Katholische Jugendsozialarbeit (BAG KJS), Düsseldorf

Wagner, S. J. (2005): Jugendliche ohne Berufsausbildung, Aachen

\section{AUTOR}

BORIS GEIER, Dr., ist Wissenschaftlicher Referent am Deutschen Jugendinstitut (DJI), München, im Forschungsschwerpunkt „Übergänge im Jugendalter". Arbeitsschwerpunkte: Berufliche Integration von sozial- und bildungsbenachteiligten Jugendlichen, Sozialberichterstattung.

geier@dji.de 